\title{
Combinatorial Models for the Finite-Dimensional Grassmannians*
}

\author{
Nicolai E. Mnëv' and Günter M. Ziegler ${ }^{2}$ \\ ${ }^{1}$ Ul. Korablestroiteley 40-1-187, \\ 199155 St. Petersburg, Russia \\ ${ }^{2}$ Institut Mittag-Leffer, \\ S-18262 Djursholm, Sweden
}

\begin{abstract}
Let $\mathscr{M}^{n}$ be a linear hyperplane arrangement in $\mathbb{R}^{n}$. We define two corresponding posets $\mathscr{G}_{k}\left(\mathscr{M}^{n}\right)$ and $\mathscr{V}_{k}\left(\mathscr{M}^{n}\right)$ of oriented matroids, which approximate the Grassmannian $G_{k}\left(\mathbb{R}^{n}\right)$ and the Stiefel manifold $V_{k}\left(\mathbb{R}^{n}\right)$. The basic conjectures are that the "OM-Grassmannian" $\mathscr{G}_{k}\left(\mathscr{M}^{n}\right)$ has the homotopy type of $G_{k}\left(\mathbb{R}^{n}\right)$, and that the "OM-Stiefel bundle" $\Delta \pi: \Delta \mathscr{V}_{k}\left(\mathscr{M}^{n}\right) \rightarrow \Delta \mathscr{G}_{k}\left(\mathscr{M}^{n}\right)$ is a surjective map. These conjectures can be proved in some cases: we survey the known results and add some new ones. The conjectures fail if they are generalized to nonrealizable oriented matroids $\mathscr{M}^{\mathbf{n}}$.
\end{abstract}

\section{Introduction}

A combinatorial model for the real Grassmannian $G_{k}\left(\mathbb{R}^{n}\right)$ arises when the "ambient space" $\mathbb{R}^{n}$ is approximated by the cell complex of a finite real hyperplane arrangement $\mathscr{M}^{n}$. For this we consider all the possible extensions of $\mathscr{M}^{n}$ by $k$-dimensional flats $V^{k}$ that are allowed to be topologically deformed, but mildly enough to make sure that the intersections of $V^{k}$ with the hyperplanes in $\mathscr{H}^{n}$ define a correct (pseudo) arrangement in $V^{k}$. This is modeled in the unit sphere $S^{n-1} \subseteq \mathbb{R}^{n}$ using the theory of pseudoarrangements and oriented matroids $\left[\mathrm{BLS}^{+}\right.$], see Section 2 below.

The combinatorial equivalence classes of extensions of the hyperplane

* Nicolai E. Mnëv was partially supported by the Institut Mittag-Leffler, Günter M. Ziegler's current address is Konrad-Zuse-Zentrum für Informationstechnik Berlin, Heilbronner Strasse 10, 1000 Berlin 31, Federal Republic of Germany. ziegler@zib-berlin.de. 
arrangement $\mathscr{M}^{n}$ by such pseudo $k$-flats form a finite poset

$$
\mathscr{G}_{k}\left(\mathscr{A}^{n}\right)
$$

whose order complex (simplicial complex of chains) serves as a combinatorial model for real Grassmannian $G_{k}\left(\mathbb{R}^{n}\right)$. The basic conjecture is that $\mathscr{G}_{k}\left(\mathscr{M}^{n}\right)$ has the homotopy type of $G_{k}\left(\mathbb{R}^{n}\right)$ for all real arrangements, that is, for all realizable oriented matroids $\mathscr{M}^{n}$.

Recent interest in such combinatorial models for real Grassmannians stems from two independent sources:

Characteristic Classes. In the case of simplicial manifolds, where a differentiable structure is not available, Gel'fand and MacPherson [GM], [Ma] suggest the following combinatorial model (which Gel'fand calls the "MacPhersonian") as a substitute for the target of the Gauss map. The MacPhersonian $\operatorname{McP}(n, k)$ is the order complex of the poset of all oriented matroids of rank $k$ on $n$ elements, ordered by weak maps. From this point of view it is interesting to find whether the cohomology of the MacPhersonian (with $\mathbb{Z} / 2$-coefficients) agrees with that of $G_{k}\left(\mathbb{R}^{n}\right)$. We see below that the MacPhersonian is in fact canonically isomorphic to $\mathscr{G}_{k}\left(\mathscr{F}^{n}\right)$, where $\mathscr{F}^{n}$ is the free oriented matroid of coordinate hyperplanes in $\mathbb{R}^{n}$. We collect some supporting evidence for the conjecture that this model has the required properties.

Extension Spaces and Semialgebraic Geometry. Consider a projection $\pi: P \rightarrow Q$ of polytopes. The finite set of polyhedral subdivisions of $Q$ that are induced by $\pi$ is partially ordered by refinement. The Generalized Baues Conjecture, as posed by Billera et al. [BKS], is that the order complex of this poset always has the homotopy type of a sphere of $\operatorname{dimension} \operatorname{dim}(P)-\operatorname{dim}(Q)-1$. The special case when $P$ is a cube (and hence $Q$ is a zonotope) is equivalent to the "extension space conjecture," stating that $\mathscr{G}_{n-1}\left(\mathscr{M}^{n}\right) \simeq \mathbb{R} P^{n-1}$ whenever $\mathscr{M}$ is realizable, see [SZ].

This conjecture is of special interest from the point of view of linear representations of oriented matroids (which is a form of first-order ordered field theory). The space $\mathscr{E}(\mathscr{M})$ can be seen as an intrinsically defined representation space of an oriented matroid. In fact, the chain complex of the poset of all embeddings of adjoints of $\mathscr{M}^{n}$ into $\mathscr{E}\left(\mathscr{M}^{n}\right)$ can be considered as a first approximation of the space of linear representations of $\mathscr{M}^{n}$. From this point of view the homotopy type of $\mathscr{E}\left(\mathscr{H}^{n}\right)$ can be a very nontrivial invariant of linear representability.

In this paper we start (Section 2) with the construction of $\mathscr{G}_{k}\left(\mathscr{M}^{n}\right)$ and a collection of partial results about its topology, which are all very recent. In Section 3 we define the $O M$-Stiefel space $\mathscr{V}_{k}\left(\mathscr{H}^{n}\right)$ over the OM-Grassmannian and present evidence for the conjecture that the projection map is always surjective. In particular, this is true in the case of the MacPhersonian, $\mathscr{M}^{n}=\mathscr{F}^{n}$.

There is an analogous treatment possible for the oriented Grassmannians, where the double cover of $\mathscr{G}_{k}\left(\mathscr{M}^{n}\right)$ is constructed by replacing every oriented 
matroid by its two possible basis orientations (chirotopes). This provides, in particular, the setting for the extension space conjecture mentioned above. To avoid technicalities we restrict our attention to the unoriented case in this paper.

We feel that the OM-Grassmannians and the OM-Stiefel bundles are both very basic objects that can unify the treatment of some crucial but delicate geometric questions. We do not claim originality: the basic idea is due to MacPherson and many of the observations in this paper are probably "folklore."

\section{The OM-Grassmannian and Its Homotopy Type}

We refer to $\left[\mathrm{BLS}^{+}\right]$for an exposition of the theory of oriented matroids. A good reference for the methods of combinatorial topology is [Bj]. Here we only review the basic set-up and fix terminology.

Let $\left\{H_{1}, \ldots, H_{m}\right\}$ be a set of $m$ hyperplanes through the origin in $\mathbb{R}^{n}$. Without loss of information we can intersect with the unit sphere $S^{n-1}$ and consider the corresponding sphere arrangement $\mathscr{A}^{n}=\left\{S_{1}^{0}, \ldots, S_{m}^{0}\right\}$ in $S^{n-1}$. Assume that the intersection $\bigcap_{i=1}^{m} S_{i}^{0}=\varnothing$ is trivial, and that a positive side $S_{i}^{+}$has been chosen for every hyperplane. Then the arrangement defines a regular cell decomposition $\Gamma\left(\mathscr{A}^{n}\right)$ of $S^{n-1}$. Every face of the cell decomposition corresponds to a covector, i.e., a sign vector $X \in\{+,-, 0\}^{m}$, where $X_{i}=+$ means that the face lies on the positive side of the $i$ th hyperplane, $X_{i}=0$ means that it lies on the $i$ th hyperplane, etc. This set $\mathscr{L} \subseteq\{t,-, 0\}^{m}$ of sign vectors satisfies the covector axioms for oriented matroids [BLS ${ }^{+}$, Section 4.1]; it is referred to as the oriented matroid $\mathscr{M}^{n}$ of rank $n$ associated with $\mathscr{A}^{n}$ (given by its covectors).

The covectors are partially ordered componentwise, where $0<+$ and $0<-$. The corresponding partially ordered set of sign vectors, also denoted by $\mathscr{L}$, is canonically isomorphic to the face poset of the arrangement (resp. of $\Gamma\left(\mathscr{A}^{n}\right)$ ). In particular, the order complex $\Delta \mathscr{L}\left(\mathscr{M}^{n}\right) \backslash 0$ is isomorphic to the barycentric subdivision $\operatorname{sd}\left(\Gamma\left(\mathscr{A}^{n}\right)\right)$. Thus the arrangement $\mathscr{A}^{n}$ can be reconstructed from the oriented matroid up to homeomorphism.

In general, the simplicial complex $\Delta \mathscr{L}$ of an oriented matroid $\mathscr{M}^{n}$ does not correspond to a real arrangement, that is, the oriented matroid is not realizable. However, $\Delta \mathscr{L}$ is the barycentric subdivision of a $P L$ regular cell decomposition $\Gamma\left(\mathscr{M}^{n}\right)$ of $S^{n-1}$ which corresponds to an arrangement of pseudospheres, i.e., images of $S^{n-2}$ under a self-homeomorphism of $S^{n-1}$ [BLS ${ }^{+}$, Section 5.1]. For general oriented matroids (as they occur in the OM-Grassmannians) it has to be allowed that the arrangements under consideration contain multiple copies of a pseudosphere, and possibly "loops," given by copies of the ambient sphere $S^{n-1}$. We refer to Section 5.1 of $\left[\mathrm{BLS}^{+}\right]$for precise definitions and for details. In all of the following $\mathscr{M}^{n}$ denotes an oriented matroid of rank $n$ on a ground set of size $m$, which we identify with the set $[m]:=\{1, \ldots, m\}$. With these precautions, the Topological Representation Theorem of Folkman and Lawrence [FL], [BLS ${ }^{+}$, Chapters 4 and 5] asserts an equivalence between

- the oriented matroids of rank $n$ on [m] (as given by their covectors), and

- the equivalence classes of arrangements of $m$ pseudospheres in $S^{n-1}$. 
If $\mathscr{M}^{n}$ and $\mathscr{N}^{k}$ are two oriented matroids on the set [m], then their relation is described as a strong map, and denoted by $\mathscr{M}^{n} \rightarrow \mathscr{N}^{k}$, if every covector of $\mathscr{N}^{k}$ is a covector of $\mathscr{K}^{n}$. Note that this condition implies $n \geq k$ [BLS ${ }^{+}$, Section 7.7].

If $\mathcal{N}_{1}^{k}$ and $\mathcal{N}_{2}^{k}$ are two oriented matroids on the set $[m]$ of the same rank, then their relation is described as a weak map, and denoted by $\mathscr{N}_{1}^{k} \leadsto \mathscr{N}_{2}^{k}$, if every covector $X_{2} \in \mathscr{L}\left(\mathcal{N}_{2}^{k}\right)$ satisfies $X_{2} \leq X_{1}$ for some covector $X_{1} \in \mathscr{L}\left(\mathcal{N}_{1}^{k}\right)\left[\mathrm{BLS}^{+}\right.$, Section 7.7].

Definition 2.1. Let $\mathscr{M}^{n}$ be an oriented matroid of rank $n$ on the set [m]. The $O M$-Grassmannian of $\mathscr{M}^{n}$ is the poset

$$
\mathscr{G}_{k}\left(\mathscr{M}^{n}\right):=\left\{\mathscr{N}^{k}: \mathscr{M}^{n} \rightarrow \mathscr{N}^{k}\right\}
$$

of all strong map images of $\mathscr{M}^{n}$ that have rank $k$, partially ordered by weak maps:

$$
\mathcal{N}_{1}^{k} \geq \mathscr{N}_{2}^{k} \Leftrightarrow \mathcal{N}_{1}^{k} \leadsto \mathcal{N}_{2}^{k}
$$

To get a geometric interpretation, we can consider $\mathscr{G}_{k}\left(\mathscr{M}^{n}\right)$ as the set of equivalence classes of embeddings $h: S^{k-1} \rightarrow S^{n-1}$ such that the intersections of the pseudospheres $S_{i}^{0}$ with $h\left(S^{k-1}\right)$ determines a pseudoarrangement in $S^{k-1}$. Two such embeddings are considered equivalent if their images intersect the same set of faces of $\mathscr{M}^{k}$ (i.e., open cells of $\Gamma\left(\mathscr{M}^{k}\right)$ ).

Thus every equivalence class of embeddings corresponds to a strong map $\mathscr{M}^{n} \rightarrow \mathscr{N}^{k}$. For the converse, note that whenever $\mathscr{M}^{n} \rightarrow \mathscr{N}^{k}$, the inclusion $\mathscr{L}\left(\mathcal{N}^{k}\right) \subseteq \mathscr{L}\left(\mathscr{M}^{n}\right)$ defines a canonical simplicial embedding of the $(k-1)$-sphere $\Delta\left(\mathscr{L}\left(\mathscr{N}^{k}\right) \backslash 0\right)$ into the topological realization $\Delta\left(\mathscr{L}\left(\mathscr{M}^{n}\right) \backslash 0\right)=\operatorname{sd} \Gamma\left(\mathscr{M}^{n}\right)$ of $\mathscr{M}^{n}$.

Now a weak map of oriented matroids corresponds to a deformation of the extension sphere into a "more special position." Thus $\Delta \mathscr{G}_{k}\left(\mathscr{M}^{n}\right)$ is in fact a topological model for the space of all extensions of $\Gamma\left(\mathscr{M}^{n}\right)$ by a pseudo $(k-1)$ sphere.

If $\mathscr{M}^{n}$ is realizable, then there is a natural map $\pi: G_{k}\left(\mathbb{R}^{n}\right) \rightarrow \mathscr{G}_{k}\left(\mathscr{M}^{n}\right)$. However, this map (the "oriented matroid stratification of $G_{k}\left(\mathbb{R}^{n}\right)$ ") is universal in the sense that even for $k=3$ and $\mathscr{K}^{n}=\mathscr{F}^{n}$ every semialgebraic variety (up to trivial stabilization) occurs as a fiber, for large enough $n$ [M]. Also the map is not in general surjective, due to the existence of nonrealizable oriented matroids. Thus, we cannot expect this map to carry a homotopy equivalence.

However, the hope is that, for realizable $\mathscr{M}^{n}$, the space $\Delta \mathscr{G}_{k}\left(\mathscr{M}^{n}\right)$ is nevertheless a good approximation to $G_{k}\left(\mathbb{R}^{n}\right)$ if $\mathscr{M}$ is "dense," and that its homotopy structure does not depend on this density condition.

Conjecture 2.2. If $\mathscr{H}^{n}$ is realizablè, then its Grassmannians are homotopy equivalent to the real Grassmannian, that is,

$$
\mathscr{G}_{k}\left(\mathscr{M}^{n}\right) \simeq G_{k}\left(\mathbb{R}^{n}\right)
$$

for all $1 \leq k \leq n-1$. 
The following two theorems explain the current status of this conjecture. The first one collects the supporting evidence that is available, the second one shows two negative results: the conjecture becomes false if only the realizable extensions of $\mathscr{M}^{n}$ are considered, and also if it is applied to the subspace of the OMGrassmannian that is spanned by realizable oriented matroids.

Theorem 2.3. Let $\mathscr{M}^{n}$ be an arbitrary oriented matroid.

(1) If $k=1$, then $\mathscr{G}_{k}\left(\mathscr{M}^{n}\right)$ is homeomorphic to $G_{1}\left(\mathbb{R}^{n}\right)=\mathbb{R} P^{n-1}$.

If $k=2$, then $\mathscr{G}_{k}\left(\mathscr{M}^{n}\right)$ is homotopy equivalent to $G_{k}\left(\mathbb{R}^{n}\right)$. In particular, it is always connected.

(2) If $k=n-1$, then $\mathscr{G}_{k}\left(\mathscr{M}^{n}\right) \cong \mathscr{E}(\mathscr{M}) / 2$ is the unoriented extension space of $\mathscr{M}^{n}$. This space is homotopy equivalent to $\mathbb{R}^{n-1}=G_{n-1}\left(\mathbb{R}^{n}\right)$

if $n \leq 3$,

if $m \leq n+2$, and

if $\mathscr{M}^{n}=\mathrm{C}^{m, n}$ is an alternating oriented matroid.

(3) If $\mathscr{M}^{n}=\mathscr{F}^{n}$ is the free oriented matroid on $m=n$ elements, then $\mathscr{G}_{k}\left(\mathscr{M}^{n}\right) \cong$ $\operatorname{McP}(n, k)$ is the MacPhersonian.

(a) If $k \leq 3$, then the MacPhersonian $\mathscr{G}_{k}\left(\mathscr{F}^{n}\right)$ is homotopy equivalent to $G_{k}\left(\mathbb{R}^{n}\right)$.

(b) For all $k$, the MacPhersonian $\mathscr{G}_{k}\left(\mathscr{F}^{n}\right)$ is connected.

Proof. (1) This is equivalent to the Topological Representation Theorem: in fact, $\mathscr{G}_{1}\left(\mathscr{M}^{n}\right)$ is the poset of all opposite pairs of nonzero covectors of $\mathscr{M}^{n}$, so $\Delta \mathscr{G}_{1}\left(\mathscr{M}^{n}\right)$ is obtained from $\Delta\left(\mathscr{L}\left(\mathscr{M}^{n}\right) \backslash 0\right)=\operatorname{sd} \Gamma\left(\mathscr{M}^{n}\right) \cong S^{n-1}$ by identification of antipodal points.

To see that $\mathscr{G}_{2}\left(\mathscr{U}^{n}\right)$ is connected we use basic facts about the "poset of regions," which can be found in Sections 4.2 and 4.4 of $\left[\mathrm{BLS}^{+}\right]$. First observe that every extension in $\mathscr{G}_{2}\left(\mathscr{M}^{n}\right)$ can be deformed to general position. Now consider two extensions $\mathscr{N}_{1}$ and $\mathscr{N}_{2}$ in general position, and choose corresponding regions (also known as maximal covectors or topes) $R_{1}, R_{2}$ of $\mathscr{M}^{n}$ which are crossed by $\mathscr{N}_{1}$ (resp. $\mathscr{N}_{2}$ ). Then $\mathscr{N}_{1}$ corresponds to a maximal chain $\mathscr{C}_{1}$ in the poset of regions $\mathscr{T}\left(\mathscr{M}^{n}, R_{1}\right)$, and $\mathscr{N}_{2}$ corresponds to a maximal chain in $\mathscr{T}\left(\mathscr{M}^{n}, R_{2}\right)$. We can choose a maximal chain $\mathscr{C}$ of $\mathscr{T}\left(\mathscr{M}^{n}, R_{1}\right)$ that contains $R_{2}$ : it will correspond to an extension $\mathscr{N}$ and also to a maximal chain in $\mathscr{T}\left(\mathscr{M}^{n}, R_{2}\right)$ that contains $R_{1}$. Now we need that the extensions in $\mathscr{G}_{2}\left(\mathscr{M}^{n}\right)$ that correspond to maximal chains in $\mathscr{T}\left(\mathscr{M}^{n}, R_{1}\right)$ are connected to each other, showing that $\mathscr{N}_{1}$ is connected to $\mathscr{N}$ in $\mathscr{G}_{2}\left(\mathscr{M}^{n}\right)$, and similarly that $\mathcal{N}$ is connected to $\mathscr{N}_{2}$. However, this follows from a lemma of Salvetti [S, Lemma 11], which is easy to generalize to oriented matroids, see Lemma 4.4 .4 of $\left[\mathrm{BLS}^{+}\right]$.

The much stronger result $\mathscr{G}_{2}\left(\mathscr{M}^{n}\right) \simeq G_{2}\left(\mathbb{R}^{n}\right)$ was announced by Babson [Ba1], [Ba2].

(2) This is equivalent to some main results from Sturmfels and Ziegler [SZ], to where we refer for details and proofs. The key fact needed for the translation to the unoriented Grassmannian, as considered here, is that the homotopy equivalences constructed in $[\mathrm{SZ}]$ are equivariant with respect to the antipodal action of $\mathbb{Z} / 2$. 
(3) This follows from the observation that, for every oriented matroid $\mathscr{M}^{k}$ on the ground set $[n]$, there is a strong map $\mathscr{F}^{n} \rightarrow \mathscr{M}^{k}$.

Part (3a) and related results will appear in Babson [Ba1]. We also refer to his work for more information about flag spaces of oriented matroids, etc.

Part (3b) follows from the observation that the minimal elements of $\operatorname{McP}(n, k)$ are exactly the oriented matroids with exactly one basis. Those are all realizable and easily seen to be connected in $M c P(n, k)$, using matroid basis exchange.

\section{Theorem 2.4 [SZ], [MR].}

(1) There is a realizable oriented matroid Suv $(14)^{3}$ of rank 3 on 14 points whose space of all realizable extensions $\mathscr{G}_{2}\left(\mathrm{Suv}(14)^{3}\right)_{\text {real }}$ is not homotopy equivalent to a sphere.

(2) There is a nonrealizable uniform oriented matroid $\mathrm{R}(19)^{4}$ of rank 4 on 19 points whose extension space contains two isolated points.

Part (2) of Theorem 2.4 means that if the extension space conjecture is true, then the extension complex gives nontrivial criteria for realizability which are of a completely new kind from a logic point of view. For each chirotope of an oriented matroid $\chi:\left(\begin{array}{l}n \\ k\end{array}\right) \rightarrow\{+,-, 0\}$ we can canonically associate the formula

$$
\Phi_{\chi}(X)=\left(\underset{J \in\left(\begin{array}{l}
n \\
k
\end{array}\right)}{\forall} \operatorname{sign}\left(\operatorname{det} X_{J}\right)=\chi(J)\right)
$$

where $X_{J}$ is a square submatrix of the variable matrix $X=\left(x_{i j}\right)_{1 \leq i \leq n, 1 \leq j \leq k}$. (By the Universality Theorem [M] every formula of first-order ordered field theory has such a form.) Consider the proposition " $\exists X: \Phi_{\chi}(X)$." The realizability problem of $\chi$ is the problem whether " $\exists X: \Phi_{\chi}(X)$ " is a theorem of first-order ordered field theory. In such a context the extension complex is a structured set of formulas which almost look like $\Phi_{\chi}$. It will be extremely interesting to understand the natural structure of such a "cloud of formulas" around $\Phi_{\chi}$, and to what extent it has a "memory" about whether " $\exists X: \Phi_{\chi}(X)$ " is a theorem or not.

\section{The OM-Stiefel Space and Its Projection}

Definition 3.1. Let $\mathscr{M}^{n}$ be an oriented matroid on [m], and let $1 \leq k \leq n-1$. The $O M$-Stiefel poset $\mathscr{V}_{k}\left(\mathscr{M}^{n}\right)$ is the set of all extensions of $\mathscr{M}^{n}$ to an oriented matroid $\hat{\mathscr{M}}^{n}$ on $[m+n-k]$ such that the set of $n-k$ "new" elements

$$
A:=\{m+1, \ldots, m+n-k\}
$$

is independent, that is, the contraction $\hat{\mathscr{M}}^{n} / A$ has rank $k$. The set $\mathscr{V}_{k}\left(\mathscr{M}^{n}\right)$ is partially ordered by weak maps. 
The chain complex $\Delta \mathscr{V}_{k}\left(\mathscr{M}^{n}\right)$, the "OM-Stiefel space," is supposed to serve as a topological model for the (noncompact) Stiefel manifold $V_{k}\left(\mathbb{R}^{n}\right)$ : the space of all ordered sets of $n-k$ linearly independent vectors, or, equivalently, of all extensions of $\mathbb{R}^{n}$ by $n-k$ linear oriented hyperplanes whose intersection has dimension $k$. Intersection of the hyperplanes induces a map $p: V_{k}\left(\mathbb{R}^{n}\right) \rightarrow G_{k}\left(\mathbb{R}^{n}\right)$. This defines the Stiefel bundle, a locally trivial fiber bundle with fiber $G L\left(\mathbb{R}^{n-k}\right)[\mathrm{H}]$. The combinatorial analogue of this bundle is given as follows.

Lemma 3.2. There is an order-preserving map to the OM-Grassmannian

$$
\pi: \mathscr{V}_{k}\left(\mathscr{M}^{n}\right) \rightarrow \mathscr{G}_{k}\left(\mathscr{M}^{n}\right), \quad \hat{\mathscr{M}}^{n} \mapsto \hat{\mathscr{M}}^{n} / A
$$

which induces a simplicial projection map

$$
\Delta \pi: \Delta \mathscr{V}_{k}\left(\mathscr{M}^{n}\right) \rightarrow \Delta \mathscr{G}_{k}\left(\mathscr{M}^{n}\right)
$$

Conjecture 3.3. For every arrangement (realizable oriented matroid) $\mathscr{M}^{n}$ the projection map

$$
\Delta \pi: \Delta \mathscr{V}_{k}\left(\mathscr{M}^{n}\right) \rightarrow \Delta \mathscr{G}_{k}\left(\mathscr{M}^{n}\right)
$$

of Lemma 3.2 is surjective for all $k$.

Conjecture 3.3 asks only the first main structural question. Of course, we would also like to know whether $\mathscr{V}_{k}\left(\mathscr{M}^{n}\right)$ is in general homotopy equivalent to the Stiefel manifold $V_{k}\left(\mathbb{R}^{n}\right)$, and about the structure of the fibers of the map $\Delta \pi$, which should approximate the group $\operatorname{GL}\left(\mathbb{R}^{n-k}\right)$.

Furthermore, it is natural to consider the OM-flag spaces

$$
\mathscr{F}\left(\mathscr{M}^{n} ; n-1, \ldots, k\right):=\left\{\left(\mathscr{M}^{n-1}, \ldots, \mathscr{M}^{k}\right): \mathscr{M}^{n} \rightarrow \mathscr{M}^{n-1} \rightarrow \cdots \rightarrow \mathscr{M}^{k}\right\}
$$

as studied by Babson [Ba1]. There is a natural map from the Stiefel space $\mathscr{V}^{k}\left(\mathscr{M}^{n}\right)$ to the OM-flag space $\mathscr{F}\left(\mathscr{M}^{n} ; n-1, \ldots, k\right)$, which is induced by successive contractions. Again, it is natural to ask whether these maps are always surjective [Ba2].

We now list the supporting evidence for Conjecture 3.3, then point out that it is not true without the realizability assumption.

\section{Theorem 3.4.}

(1) If $k=1$, then $\Delta \pi: \Delta \mathscr{V}_{k}\left(\mathscr{M}^{n}\right) \rightarrow \Delta \mathscr{G}_{k}\left(\mathscr{M}^{n}\right)$ is surjective.

(2) If $k=n-1$, then $\pi$ : $\mathscr{V}_{k}\left(\mathscr{M}^{n}\right) \cong \mathscr{G}_{k}\left(\mathscr{M}^{n}\right)$ is an isomorphism of posets.

(3) If $\mathscr{M}^{n}=\mathscr{F}^{n}$, then $\Delta \pi: \Delta \mathscr{V}_{k}\left(\mathscr{M}^{n}\right) \rightarrow \Delta \mathscr{G}_{k}\left(\mathscr{M}^{n}\right)$ is surjective. 
Proof. (1) For this, lexicographic extensions can be used [BLS ${ }^{+}$, Section 7.2]. In fact, every maximal simplex of $\mathscr{G}_{1}\left(\mathscr{M}^{n}\right)$ can be represented by a maximal flag $0<$ $Y^{1}<\cdots<Y^{n}$ of covectors in $\mathscr{L}=\mathscr{L}\left(\mathscr{M}^{n}\right)$. After relabeling and reorientation we may assume that the $Y^{s}$ are determined by the basis $\{1,2, \ldots, n\}$ of $\mathscr{M}^{n}$ via

$$
Y_{i}^{s}= \begin{cases}0 & \text { if } \quad i \in\{s+1, \ldots, n\} \\ + & \text { if } \quad i \in\{1, \ldots, s\}\end{cases}
$$

Now we construct lexicographic extensions

$$
\begin{aligned}
& \hat{\mathscr{M}}_{1}^{n}:=\mathscr{M}^{n}+\left[e_{2}^{+}\right]+\left[e_{3}^{+}\right]+\cdots+\left[e_{n}^{+}\right], \\
& \hat{\mathscr{M}}_{2}^{n}:=\mathscr{M}^{n}+\left[e_{2}^{+}, e_{1}^{-}\right]+\left[e_{3}^{+}\right]+\cdots+\left[e_{n}^{+}\right] \text {, } \\
& \hat{\mathscr{M}}_{n}^{n}:=\mathscr{M}^{n}+\left[e_{2}^{+}, e_{1}^{-}\right]+\left[e_{3}^{+}, e_{1}^{-}\right]+\cdots+\left[e_{n}^{+}, e_{1}^{-}\right] .
\end{aligned}
$$

To see that this yields the required result, first observe two facts:

(a) If $e_{1}$ and $f_{1}$ are different and not parallel, then the lexicographic extensions $p=\left[e_{1}^{\alpha_{1}}, \ldots, e_{k}^{\alpha_{k}}\right]$ and $p^{\prime}=\left[f_{1}^{\beta_{1}}, \ldots, f_{l}^{\beta_{i}}\right]$ commute:

$$
\mathscr{M}^{n}+p+p^{\prime}=\mathscr{M}^{n}+p^{\prime}+p
$$

(b) For $k \geq l$, there is a weak map between lexicographic extensions

$$
\mathscr{M}^{n}+\left[e_{1}^{\alpha_{1}}, \ldots, e_{k}^{\alpha_{k}}\right] \leadsto \mathscr{M}^{n}+\left[e_{1}^{\alpha_{1}}, \ldots, e_{l}^{\alpha_{l}}\right]
$$

From this we get that there is a sequence of weak maps $\hat{\mathscr{M}}_{n}^{n} \leadsto \cdots \cdots \rightarrow \hat{\mathscr{M}}_{2}^{n} \leadsto \rightarrow \hat{\mathscr{M}}_{1}^{n}$. Furthermore, by induction on $s$ it follows that the extension hyperplanes of $\hat{\mathscr{M}}_{s}^{n}$ intersect in $\pm Y^{s}$. This proves that the simplex $\left[ \pm Y^{n}, \ldots, \pm Y^{2}, \pm Y^{1}\right]$ is in the image of $\Delta \pi$, with

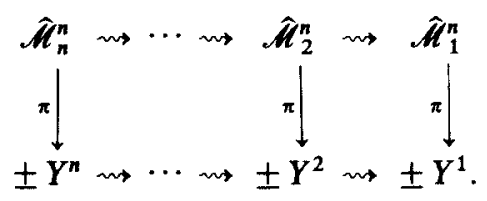

(2) This is an immediate consequence of the embedding into the first barycentric subdivision, as discussed after Definition 2.1, see Exercise 7.30 of [BLS ${ }^{+}$].

(3) That the map $\pi: \mathscr{V}_{k}\left(\mathscr{H}^{n}\right) \rightarrow \mathscr{G}_{k}\left(\mathscr{H}^{n}\right) \cong M c P(n, k)$ is surjective follows from Brylawski and Ziegler [BZ, Theorem 2]. In fact, the construction " $\mathscr{M}^{n} \rightarrow \hat{\mathscr{M}}^{n} \backslash A$ " given there describes an explicit section. To see that in fact $\Delta \pi$ is surjective, we can use a simpler variant of the construction from [BZ], which is not concerned about the complementary minor; in particular, we avoid the use of unions, which in general do not preserve weak maps. 
Let $\mathscr{M}_{1}^{k} \leadsto \mathscr{M}_{2}^{k} \leadsto \cdots \leadsto \mathscr{H}_{t}^{k}$ be a chain of oriented matroids of rank $k$ on the set [n]. We assume, after suitable relabeling, that $[k]$ is a basis of $\mathscr{M}_{t}^{k}$, and thus of all $\mathscr{M}_{i}^{k}$. (This choice corresponds to the selection of a suitable Schubert cell in $\mathscr{G}_{k}\left(\mathscr{M}^{n}\right)$, over which we construct a section.)

For each $j$, consider the dual oriented matroid $\left(\mathscr{M}_{j}^{k}\right)^{*}$, for which $\{k+1, \ldots, n\}$ is a basis. Then let $\left(\mathscr{M}_{j}^{k}\right)^{*}+B$ denote its extension to

$$
E=[n] \cup B:=[n] \cup\{n+k+1, \ldots, 2 n\}
$$

in such a way that $i$ is parallel to $i-n$, for all $i \in B$. We claim that the dual $\tilde{\mathscr{M}}_{j}^{k}:=\left(\left(\mathscr{M}_{j}^{k}\right)^{*}+B\right)^{*}$ has the required properties. In fact, by construction $\tilde{\mathscr{M}}_{j}^{k}$ is an oriented matroid of rank $n$ on the ground set $[n] \cup B$. If $\mathscr{M}_{j}^{k}$ is represented by a matrix $\left(I C_{j}\right)$, then $\tilde{M}_{j}^{k}$ is represented by

$$
\left(\begin{array}{ccc}
I & C_{j} & 0 \\
0 & -I & I
\end{array}\right)
$$

Furthermore,

$$
\tilde{M}_{j}^{k} / B=\left(\left(\mathscr{M}_{j}^{k}\right)^{*}+B\right)^{*} / B=\left(\left(\left(\mathscr{H}_{j}^{k}\right)^{*}+B\right) \backslash B\right)^{*}=\left(\mathscr{M}_{j}^{k}\right)^{* *}=\mathscr{M}_{j}^{k}
$$

and

$$
\tilde{\mathscr{H}}_{j}^{k} \backslash B=\left(\left(\mathscr{M}_{j}^{k}\right)^{*}+B\right)^{*} \backslash B=\left(\left(\left(\mathscr{M}_{j}^{k}\right)^{*}+B\right) / B\right)^{*}=(\text { loops on }[n])^{*}=\mathscr{F}^{n}
$$

Finally, $\tilde{\mathscr{M}}_{j}^{k}$ was constructed by parallel extensions and by duality. Both operations preserve weak maps, and hence we get

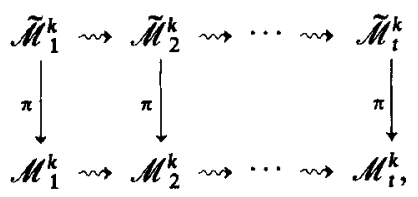

so that the simplex $\left[\mathscr{M}_{1}^{k}, \ldots, \mathscr{M}_{t}^{k}\right] \in \Delta \mathscr{G}_{k}\left(\mathscr{F}^{n}\right)$ is in the image of $\Delta \pi$.

Theorem 3.5 [R]. There is an oriented matroid $\mathrm{R}(12)^{4}$ of rank 4 on 12 points with an extension of rank 2 that is not in the image of the projection map

$$
\pi: \mathscr{V}_{2}\left(\mathrm{R}(12)^{4}\right) \rightarrow \mathscr{G}_{2}\left(\mathrm{R}(12)^{4}\right)
$$

More geometrically, the example of Theorem 3.5 asserts the following. The oriented matroid $\mathrm{R}(12)^{4}$ can be represented by an arrangement of pseudo 2 -spheres in $S^{3}$. This arrangement can be extended by a pseudo 1 -sphere $h\left(S^{1}\right)$ with the following property: there is no extension of this arrangement by a pseudosphere 
$k\left(S^{2}\right)$ that intersects all the regions of $\operatorname{MR}(12)^{4}$ which are intersected by $h\left(S^{1}\right)$. Assuming that we use the canonical embeddings into the barycentric subdivision (so that $h\left(S^{1}\right)$ is not knotted in $S^{\mathbf{3}}$ ) this is equivalent to saying that there is no embedding of $S^{2}$ so that $k\left(S^{2}\right) \supseteq h\left(S^{1}\right)$.

In particular, this kills an old conjecture of Las Vergnas (from 1975, see [AL] and Conjecture 7.7 .4 of $\left[\mathrm{BLS}^{+}\right]$) that every strong map of oriented matroids can be factored into an extension followed by a contraction. In our terminology this is equivalent to the conjecture that the projection map of Lemma 3.2 is always surjective on the set of vertices, which Theorem 3.5 disproves. In the case of ordinary matroids this statement, the "Higgs factorization theorem," is true, although nontrivial $[\mathrm{K}]$.

\section{Acknowledgments}

We gratefully acknowledge helpful conversations with Robert D. MacPherson, Eric Babson, and Bernd Sturmfels on topics related to this paper.

\section{References}

[AL] L. Allys and M. Las Vergnas: Minors of matroid morphisms, J. Combin. Theory Ser. B (to appear).

[Ba1] E. K. Babson: A combinatorial flag space, Ph.D. Thesis, MIT, 1993.

[Ba2] E. K. Babson: Personal communication.

[BKS] L. J. Billera, M. M. Kapranov, and B. Sturmfels: Cellular strings on polytopes, Proc. Amer. Math. Soc., to appear.

[Bj] A. Björner: Topological methods, in: Handbook of Combinatorics (R. Graham, M. Grötschel, and L. Lovász, eds.), North-Holland, Amsterdam, to appear.

[BLS $\left.{ }^{+}\right]$A. Björner, M. Las Vergnas, B. Sturmfels, N. White, and G. M. Ziegler: Oriented Matroids, Encyclopedia of Mathematics, Cambridge University Press, Cambridge, 1993.

[BZ] T. H. Brylawski and G. M. Ziegler: Topological representation of dual pairs of oriented matroids, Discrete Comput. Geom., this issue, pp. 237-240.

[FL] J. Folkman and J. Lawrence: Oriented matroids, J. Combin. Theory Ser. B 25 (1978), 199-236.

[GM] I. M. Gel'fand and R. D. MacPherson: A combinatorial formula for the Pontrjagin classes, Bull. Amer. Math. Soc. 26 (1992), 304-309.

[H] D. Husemoller: Fiber Bundles, McGraw-Hill, New York, 1966.

[K] J. P. S. Kung: Strong maps, in: The Theory of Matroids (N. White, ed.), Cambridge University Press, Cambridge, 1986, pp. 224-253.

[Ma] R. D. MacPherson: Combinatorial differential manifolds, Preprint, 1992.

[M] N. E. Mnëv: The universality theorems on the classification problem of configuration varieties and convex polytopes varieties, in: Topology and Geometry - Rohlin Seminar (O. Ya Viro, ed.), Lecture Notes in Mathematics, Vol. 1346, Springer-Verlag, Berlin, 1988, pp. 527-544.

[MR] N. E. Mnëv and J. Richter-Gebert: Two constructions of oriented matroids with disconnected extension space, Discrete Comput. Geom., this issue, pp. 271-285.

[R] J. Richter-Gebert: Oriented matroids with few mutations, Discrete Comput. Geom., this issue, pp. 251-269.

[S] M. Salvetti: Topology of the complement of real hyperplanes in $\mathbb{C}^{N}$, Invent. Math. 88 (1987), 603-618.

[SZ] B. Sturmfels and G. M. Ziegler: Extension spaces of oriented matroids, Discrete Comput. Geom. 10(1) (1993), 23-45.

Received December 23, 1991. 